\title{
ANÁLISE DA IMPLEMENTAÇÃO DE UM PROGRAMA DE TRANSPLANTE INTESTINAL E MULTIVISCERAL NO HOSPITAL DAS CLÍNICAS DA FACULDADE DE MEDICINA DA UNIVERSIDADE DE SAO PAULO - SISTEMA ÚNICO DE SAÚDE, ATRAVÉS DA METODOLOGIA PMBOK
}

\author{
Analysis on implementation of an intestinal and multivisceral transplantation program at Hospital \\ das Clinicas, School of Medicine, University of São Paulo - unique health system by using the \\ PMBOK methodology
}

Andre Ibrahim David', Fabio Luis Alves², Luiz Augusto Carneiro D’Albuquerque ${ }^{1}$

\section{RESUMO}

Objetivo: Analisar, com uma metodologia estabelecida, o Project Management Body of Knowledge (PMBOK), a implantação do Serviço de Transplante Intestino e Multivisceral no HC-USP, que tem um projeto de parceria do Sistema Nacional de Transplantes (SNT) e do SUS. Materiais e Métodos: Através da ferramenta de análise metodológica, PMBOK, foi realizado o estudo de cada etapa de implantação do projeto (Programa), com um olhar crítico em cada fase (Integração, Escopo, Tempo, custo, Qualidade, Recursos Humanos, Comunicações, Riscos, aquisições e interessados). Analisar se as medidas necessárias foram tomadas corretamente (os já realizados) e identificar áreas de melhoria nessas tarefas que ainda não foram realizadas. Análise de grupos de processos: Iniciação, Planejamento, Execução, Monitoramento, Controle e Encerramento. Resultados: Os resultados foram analisados utilizando os conceitos descritos e mostrados na tabela. Conclusão: A análise, de acordo com os conhecimentos adquiridos e os resultados da avaliação de cada item, mostrou que os grupos de trabalho de cada processo e suas áreas de atuação foram correlacionados de forma adequada durante todo o projeto.

Descritores: Transplante de Órgãos, Gestão em Saúde, Intestinos

Instituição:

\footnotetext{
${ }^{1}$ Departamento de Gastroenterologia, Disciplina de Transplante de Fígado e Órgãos do Aparelho Digestivo da FMUSP.

${ }^{2}$ Instituto de Ensino e Pesquisa do Hospital Sírio-Libanês, Fundação Dom Cabral
}

\author{
Correspondência: \\ Andre Ibrahim David \\ Rua Pamplona, 1808, apto 52, CEP 01405-002, Sao Paulo/SP, Brasil. \\ Fone: (11) 2597-5297 \\ E-mail: andredavidmd@gmail.com
}

Recebido em: 10.12.2012

Aceito em: 11/01/2013

\section{INTRODUÇÃO}

Atualmente, o transplante de intestino é a única possibilidade de cura para pacientes com falência intestinal que desenvolvem complicações graves relacionadas ao uso prolongado da nutrição parenteral. Estima-se que 200 pessoas por ano tenham indicação para realização de transplante de intestino no nosso país. Apesar dos avanços tecnológicos e das necessidades clínicas, ainda não há centros especializados em transplante intestinal no Brasil.

O transplante restabelece a capacidade nutricional por via oral em pacientes com falência intestinal (FI) irreversível. Historicamente prejudicado pelo sucesso inicial limitado, o transplante de intestino (TI) evoluiu de forma semelhante aos outros transplantes de órgãos e transformou-se de um procedimento experimental a uma opção terapêutica. ${ }^{1,2}$

O TI é a "última fronteira" do maior Programa de Transplantes Públicos do Mundo: o SUS. 
O governo federal investiu recentemente quase 10 milhões de reais no HCFMUSP, em dois Projetos: um que visa o suporte financeiro para o tratamento da Insuficiência Intestinal e outro que visa o apoio financeiro àqueles 10 a $15 \%$ casos em que há a necessidade do TI.

\section{Falência Intestinal}

A FI é uma condição clínica caracterizada pela redução da capacidade funcional do trato gastrointestinal para manter as condições mínimas necessárias para a digestão e absorção dos nutrientes e fluidos necessários para a manutenção nos adultos e/ou para o crescimento nas crianças.

Há apenas cinquenta anos, a FI era considerada incompatível com a vida. Com o desenvolvimento da nutrição parenteral (NPT) e mais recentemente, o transplante de intestino, houve um aumento no potencial de sobrevida e na melhora da qualidade de vida destes pacientes.

Estima-se que 1-3 pessoas por milhão de habitantes por ano apresentarão FI. Desses, 15\% serão candidatos ao transplante intestinal/multivisceral, por irreversibilidade da FI. Na infância, a FI ocorre em cerca de dois a seis/oito indivíduos a cada 1000000 de crianças em países desenvolvidos. ${ }^{3}$

\section{Etiologia da Falência Intestinal}

A perda da capacidade de absorção entérica seja de forma aguda ou crônica causada pela síndrome do intestino ultracurto (SIC), alteração na motilidade intestinal ou disfunção dos enterócitos pode levar à falência intestinal. ${ }^{4}$

Cerca de $60 \%$ dos casos ocorrem na população pediátrica. As causas mais frequentes são: a enterocolite necrotizante, gastrosquise, atresia intestinal, vólvulos, pseudo-obstrução e aganglianose. Entre os adultos, a isquemia, doenças inflamatórias (ex. Doença de Crohn), traumas e tumores são as causas mais comuns..$^{5-8}$

\section{Evolução Clínica da Falência Intestinal}

A evolução clínica da FI é de difícil prognóstico e está associada a alguns fatores de risco que levam ao uso contínuo da nutrição parenteral NP. Nas crianças, a presença da síndrome do intestino ultra curto $(<20-30 \mathrm{~cm}$ de intestino) associado à alteração de motilidade residual, perda parcial do cólon e a ausência da válvula íleocecal estão associadas à NP por longo prazo. ${ }^{9,10}$

Nos adultos, a ressecção intestinal que resulta em menos de $100 \mathrm{~cm}$ de jejuno-íleo, associada às anormalidades da mucosa restante e à ausência de válvula íleocecal, são fatores preditivos de dependência crônica de NP. Em estudo multicêntrico no Estado de São Paulo, Bakonyi e colaboradores avaliaram 248 pacientes que foram submetidos a algum tipo de ressecção intestinal em sete unidades de terapia intensiva de hospitais universitários. Observaram que 24 apresentaram SIC necessitando NPT e que cinco apresentavam indicação para transplante de intestino pelos critérios internacionais. Dos pacientes com indicação de TI, apenas dois permaneciam vivos quando a pesquisa foi concluída. ${ }^{4}$

\section{Nutrição Parenteral}

Introduzida por Dudrick e colaboradores em 1968, NP logo tornou-se a primeira linha de tratamento para muitas doenças gastrointestinais. Estima-se que cerca de 40 mil indivíduos recebam NP de forma crônica nos EUA. ${ }^{11}$

Com o sucesso da NP, as morbidades relacionadas ao seu uso prolongado tornaram-se mais frequentes. Cerca de $50 \%$ dos pacientes utilizando NP domiciliar necessitam hospitalização. A complicação mais frequente (cerca de $75 \%$ ) é a infecção relacionada ao uso do cateter. O uso da NP está associado a possíveis complicações em longo prazo. ${ }^{11}$

\section{Resultados do TI}

A sobrevivência precoce global do paciente e do enxerto após o transplante intestinal melhorou significativamente nos últimos 10 anos. Os resultados mais recentes mostram mais de 2000 destes transplantes realizados em mais de 60 centros mundiais, sendo que $50 \%$ dos receptores estão vivos e a maior parte independente da NPT. ${ }^{10}$

Em 1998, a sobrevida do enxerto e do paciente para um ano era de $52 \%$ e $69 \%$, respectivamente, enquanto em 2007, a sobrevida aumentou para $75 \%$ e $79 \%$, respectivamente, um aumento de mais de 20 pontos percentuais para a sobrevida do enxerto. ${ }^{10}$

A maioria dos pacientes apresenta boa função do enxerto e completo restabelecimento da alimentação por via oral. Esses resultados são similares aos de outros transplantes de órgãos sólidos, como o pâncreas, rim e fígado. O grupo da Universidade de Pittsburgh, que até maio de 2009 havia realizado 524 transplantes de intestino, mostra sobrevivência do paciente para um e cinco anos de 93 e 78\% respectivamente. ${ }^{2}$

Acredita-se que o aperfeiçoamento da técnica cirúrgica, imunossupressão com indução perioperatória por meio de anticorpos anti-linfocitários; controle da infecção com monitoramento específico de agentes virais como o EpsteinBarr, CMV e o adenovírus; o aumento da experiência clínica (instituições com mais de 10 transplantes realizados); a melhor compreensão da fisiopatologia do TI e das necessidades dos receptores e os avanços na detecção e tratamento de rejeição sejam os fatores mais importantes para a melhoria dos resultados.

\section{Dieta após TI}

Várias etapas são necessárias para que ocorra a autonomia nutricional após o TI.

Os pacientes são mantidos inicialmente pela NPT com lenta transição para alimentação enteral. Uma fórmula enteral é introduzida entre o $3^{\circ}$ e $14^{\circ}$ dia pós-operatório (PO) e a alimentação oral é iniciada por volta do $7^{\circ}$ ao $14^{\circ}$ dia PO.12

Geralmente, são utilizadas fórmulas com baixo teor de gordura nas primeiras semanas e a introdução dos lipídios dá-se por volta de um mês após o transplante. Quando as necessidades calóricas são alcançadas com a alimentação e a fórmula enteral, a NPT é descontinuada. ${ }^{12}$ 


\section{Qualidade de Vida e TI}

O objetivo do transplante vai além de assegurar a vida do doente. Busca-se oferecer o estado de saúde antes da doença, o equilíbrio entre a eficácia funcional do enxerto, os aspectos psicológicos e a integridade física do transplantado. O tratamento não se limita à sobrevivência e ao tempo de sobrevida; também deve-se considerar como isso afetará a qualidade de vida do paciente. A sobrevida dos pacientes submetidos ao TI tem aumentado com os avanços já descritos e a avaliação da qualidade de vida desses indivíduos começa a ser estudada. ${ }^{13}$

É evidente que a realização de um procedimento de tal porte com vários cuidados pós-transplante, implica em grande demanda tanto dos pacientes como dos familiares. Entretanto, apesar dos efeitos adversos inerentes ao tratamento, como a terapia de imunossupressão, a possibilidade de rejeição, o estresse psicossocial e custo financeiro, os resultados indicam efeitos positivos na qualidade de vida destes indivíduos. ${ }^{13}$

Em um estudo realizado por Sudan e colaboradores (em uma população pediátrica submetida ao transplante de intestino, após cinco anos de cirurgia) as crianças transplantadas sentiam-se da mesma forma que seus colegas de escola quanto aos aspectos físicos e psicossociais. Já, os pais consideravam que seus filhos apresentavam um estado de saúde pior do que seus pares. ${ }^{14}$

Entre adultos transplantados, os escores da qualidade de vida melhoram ao longo do tempo. Apesar da necessidade do uso de medicamentos, redução da mobilidade física, presença de dor e desconforto, foi descrita melhora física, social e emocional. ${ }^{13}$

\section{Da Instituição}

\section{Gestão Atual}

No passado, foram realizados seis transplantes intestinais no Brasil. O Hospital das Clínicas da Faculdade de Medicina da Universidade de São Paulo (HC-FMUSP) foi pioneiro mundial do TI, realizados pelo Professor Okumura na década de 60 (Okumura et al., 1969; Okumura \& Mester, 1992). Três outras instituições realizaram mais recentemente quatro transplantes intestinais, contudo os resultados foram desapontadores com óbito precoce de todos os receptores. ${ }^{15-17}$

Com a união atual das Disciplinas do Departamento de Gastroenterologia do Hospital das Clinicas de Sao Paulo, Gastroenterologia Clínica (Prof. Flair José Carrilho), Cirurgia do Aparelho Digestivo e Coloproctologia (Prof. Ivan Cecconello) e Transplante de Órgãos do Aparelho Digestivo (Prof. Luiz Augusto Carneiro D'Albuquerque) e com intensa dedicação institucional à FMUSP, ampliou-se o ciclo virtuoso de evolução no ensino, pesquisa e assistência. Todos os seus Professores Titulares participam hoje de Conselhos e Comissões da FMUSP e do Hospital das Clínicas, bem como outros membros do Departamento.

O Departamento de Gastroenterologia profissionalizou a gestão administrativa, contratando administradores hospitalares formados pelo PROHASA. A eficiência administrativa tem se traduzido em melhoria da assistência, da sua infraestrutura e na sistematização da captação de recursos junto aos órgãos governamentais. Nessa linha, com o auxílio importante do Prof. Sérgio Carlos Nahas, citam-se as reformas do centro cirúrgico e do ambulatório da Cirurgi, e dos Transplantes de Órgãos do Aparelho Digestivo, bem como sua informatização. Foram estabelecidos protocolos de seguimento para as principais doenças do aparelho digestivo. A reforma das salas para Transplantes do Aparelho Digestivo está sendo iniciada, o que permitirá um ganho de escala ainda maior na realização dessas operações.

Decorrente do seu crescimento científico, o Departamento investiu grandes esforços na reestruturação física e de recursos humanos nos seus LIMs, tornando-os base fundamental para a pesquisa translacional. Para tanto, importante foi a colaboração dos órgãos públicos de fomentos e da iniciativa privada, tornando-os muito produtivo.

Importante foi a incorporação da UTI do $9^{\circ}$ andar pelas Disciplinas do Departamento de Gastroenterologia, possibilitando que, em 2009, o Serviço de Transplantes de Órgãos realizasse 152 transplantes, sendo 140 de fígado e recebesse o Prêmio Destaque em Transplantes, da Secretaria de Estado da Saúde de São Paulo, como maior programa de transplantes do Brasil.

Visando a internacionalização das pesquisas do Departamento, convênios oficiais foram estabelecidos com Universidades do exterior: Barcelona (Espanha), Keio (Japão), e Chicago (EUA).

\section{Equipe}

A Disciplina de Transplante e Cirurgia do Fígado e o Instituto da Criança contam com equipes multidisciplinares exclusivamente envolvidas com atividades de transplante, formada por cirurgiões, clínicos, endoscopistas, intensivistas, infectologistas, radiologistas, patologistas, coordenadores de captação de órgãos, psicólogas, fisioterapeutas, farmacêuticos, nutricionistas, enfermeiros, pessoal administrativo, dentre outros profissionais.

Os médicos que atuarão no projeto receberam treinamento especializado em transplante intestinal e multivisceral em grandes centros internacionais (Pittsburgh, Indianápolis e Miami).

\section{Passado recente e perspectivas futuras}

O Serviço de Transplante de Fígado e Órgãos abdominais tem sido um dos três maiores do Brasil nos ultimos cinco anos, sendo inclusive, um dos únicos que realiza o transplante de fígado intervivos entre adultos no Brasil.

Estima-se que 200 pessoas por ano tenham indicação para realização de transplante de intestino no nosso país. Apesar dos avanços tecnológicos e das necessidades clínicas, ainda não há centros especializados em transplante intestinal no Brasil.

Será realizado estudo antes e depois em um grupo de dez pacientes, que serão alocados conforme a demanda clínica para realização de transplante de intestino ou transplante multivisceral. 
Foi constituído um protocolo clínico embasado no estado da arte do transplante intestinal. Esperamos alcançar resultados compatíveis com os centros de excelência mundiais que vêm realizando este procedimento de maneira rotineira. Os resultados deste estudo terão grande impacto no tratamento da falência intestinal crônica no nosso meio, oferecendo novas perspectivas terapêuticas. Além do benefício social e clínico para os pacientes afetados e suas famílias, bem como vantagens econômicas, este estudo cria oportunidades para o aprendizado e para o d0esenvolvimento de um programa de transplante de intestino no Sistema Único de Saúde.

\section{REFERENCIAL TEÓRICO - Situação Objetiva}

Pelo menos vinte casos por ano de portadores de falência intestinal são referidos ao Serviço de Transplante de Órgãos Abdominais do HC-FMUSP. Esta população de doentes tem uma mortalidade de $90 \%$ em 5 anos.

Por que ainda não fazemos Transplante de Intestino? E por que deveríamos fazê-los:

\section{Causas}

- Nunca houve investimento nessa área de atuação/subespecialidade, até janeiro de 2011 por parte do Governo e/ ou Privado.

- Ignorância da comunidade médica a respeito dessa possibilidade de tratamento.

- Alto custo do tratamento/doente.

- Falta de mão de obra multidisciplinar especializada.

- Resultados não muito satisfatórios no exterior até 2010.

- Competição com o tratamento de suporte (já consagrado) da falência intestinal: Nutrição Parenteral Total (NPT).

- Baixo nível sócio-econômico dos doentes e familiares.

\section{Consequências}

- Nunca houve investimento nessa área de atuação/subespecialidade, até Janeiro 2011 por parte do Governo e/ou Privado.

- Ignorância da comunidade médica a respeito dessa possibilidade de tratamento.

- Alto custo do tratamento/doente.

- Qualidade de vida muito ruim com a NPT, principalmente para o doente, mas também para os familiares.

- Falta de mão de obra multidisciplinar especializada.

- Resultados não muito satisfatórios no exterior, até 2010.

- Competição com o tratamento de suporte (já consagrado) da falência.

\section{Dificuldades perenes ao longo do tempo}

- Falta de investimento nessa área.

- Alto custo do tratamento/doente.

- Baixo nível sócio-econômico dos doentes e familiares.

\section{Quem são os ATORES SOCIAIS?}

Ministro da Saúde, Secretário Estadual da Saúde, Secretário Municipal de Saúde, Diretor do HC-FMUSP, Diretor da FMUSP, Prof. Titular Gastroenterologia, Prof. Titular Transplante, Prof. Nutrologia, Coordenador dos Transplantes, Equipe de Cirurgiões, Equipe Nutrologia, Coordenadora de Enfermagem, Equipe de Enfermagem, Equipe de Psicologia, Pacientes, Residentes, Imprensa, Familiares e Associação Brasileira de Transplante de Órgãos.

\section{REFERENCIAL PRÁTICO}

Em 2000, o autor participou da equipe liderada pelos Drs. Wangles Soler e Mauricio Iasi, que realizou o primeiro transplante de intestino pediátrico do Brasil e até hoje o de melhor sobrevivência (cem dias).

Realizou o mestrado (2001-2003) em Medicina (Cirurgia) pela Faculdade de Ciências Médicas da Santa Casa de São Paulo, desenvolvendo um modelo de treinamento para transplante de pâncreas em cães, modelo este que permitiu a capacitação da equipe para a era clínica daquele transplante, com a realização de cinco transplantes duplos em seres humanos. Tese orientada pelo Dr. Luiz Arnaldo Szutan e co-orientada pelo Dr. Adhemar Pacheco Junior, defendida em 2003. Adquiriu respeitabilidade nessa área de atuação, tendo sido eleito para o Departamento de Transplante de Pâncreas da ABTO (2004-2005). Programa este interrompido devido ao estágio de doutorado no exterior.

Em 2003, estagiou por dois meses no Serviço de Transplantes Adulto e Pediátrico da Universidade de Pittsburgh, onde trabalhou com os Drs. Jorge Reyes, John Fung, George Mazariegos, Sindhi Rakeshi e Paulo Fontes.

Recebeu o título de membro titular da Associação Brasileira de Transplante de Órgãos (ABTO). Realizou prova didática e recebeu o título de professor instrutor da Faculdade de Ciências Médicas da Santa Casa de São Paulo.

Realizou o doutorado-sanduíche na Universidade de Miami, serviço do Dr. Andreas Tzakis, com vínculo da pós-graduação da Faculdade de Ciências Médicas da Santa Casa de São Paulo e bolsa do CNPq, em transplante de intestino delgado e multivisceral e em células mesenquimais (2004-2007). Nesse período, pode atuar tanto nas pesquisas com células mesenquimais e transplante de intestino em ratos quanto nas atividades clínico-cirúrgicas do Programa de Transplante Hepático e Intestinal, onde participou ativamente de 50 transplantes intestinais e multiviscerais tanto em adultos como em crianças. Nesse período realizou a coleta de dados da tese de doutorado, que encaminhou para publicação, tendo sido aceita e publicada na Transplantation. Além desse, participou ativamente de inúmeros trabalhos do Serviço do Dr. Andreas Tzakis que foram publicados em revistas Qualis A e B nos anos de 2006 e 2007.

A tese de doutorado foi orientada pelo Dr. Andreas G. Tzakis e coorientada pelo Dr. Luiz Arnaldo Szutan, tendo sido defendida no Brasil, em 2007.

Membro aceito pela American Society of Transplant Surgeons como membro internacional (2006). 


\section{OBJETIVOS}

\section{Geral:}

O objetivo deste estudo é analisar com uma metodologia estabelecida Project Management Body of Knowledge (PMBOK) (18) a implementação do Serviço de Transplante de Intestino no HC-FMUSP, projeto que tem a parceria do Sistema Nacional de Transplantes (SNT) e do SUS.

\section{Específicos:}

Identificar possíveis falhas na elaboração do projeto e da sua implementação.

1. Analisar os processos de cada grupo de trabalho.

\section{MÉTODOS}

Através da ferramenta de análise metodológica, a Project Management Body of Knowledge (PMBOK - tabela 1) realizamos o estudo de cada etapa da Implementação do Projeto (Programa), com um olhar crítico em cada fase (Integração, Escopo, Tempo, Custos, Qualidade, Recursos Humanos, Comunicação, Riscos, Aquisição e Partes Interessadas), e se as medidas necessárias foram tomadas de maneira correta (naquelas já realizadas), identificando pontos de melhoria naquelas tarefas que ainda não foram realizadas.

Áreas de conhecimento:

1. Gerenciamento da Integração do projeto (desenvolver o termo de abertura do projeto)

2. Gerenciamento do escopo do projeto

3. Gerenciamento do tempo do projeto

4. Gerenciamento dos custos do projeto

5. Gerenciamento de qualidade do projeto

6. Gerenciamento dos recursos humanos do projeto

7. Gerenciamento das comunicações do projeto

8. Gerenciamento dos riscos do projeto

9. Gerenciamento das aquisições do projeto

10.Gerenciamento das partes interessadas do projeto

Grupo de Processos:

1. Iniciação

2. Planejamento

3. Execução

4. Monitoramento e controle

5. Encerramento
Nessas tabelas, foram analisados, de acordo com os conceitos do $\mathrm{PMBOK} 5^{\circ}$ edição, os resultados práticos e reais da implementação do Projeto. Em seguida, foram dadas notas médias por cada Grupo de processo, conforme abaixo:

\section{Notas:}

A - Relação forte da teoria com a realidade

B - Relação fraca da teoria com a realidade

C - Nenhuma relação da teoria com a realidade

\section{RESULTADOS}

Os resultados foram analisados utilizando os conceitos descritos na Tabela 1.

\section{DISCUSSÃO}

A iniciação do projeto foi realizada com ampla discussão entre as partes interessadas. Além dos atores já citados, ainda estendeu-se a discussão para áreas afins do serviço no Hospital, como Banco de Sangue e Laboratório de Investigações Médicas, o LIM-37. O Escopo e a Estrutura Analítica do Projeto foram exaustivamente pensados e repensados entre os idealizadores do Projeto.

O Planejamento foi adequado mediante as necessidades identificadas no Projeto. Houve adequações e readequações ao planejamento inicial, devido a mudanças de recursos humanos (repatriação de colega do exterior) e de reforma das unidades de internação (Centro-cirúrgico, UTI e enfermaria).

A execução foi realizada passo a passo. Como havia o prévio amplo desenvolvimento das descrições técnicas protocolares dos processos operacionais (Anexo 1 e 2) e a redação prévia do extenso Protocolo de Transplante de Intestino e Multivisceral em conjunto com as equipes médicas especialistas (Planejamento), a Execução foi gradual ao longo de dois anos e meio. Ocorreu ida dos profissionais a estágios em grandes Centros de Transplante Intestinal no exterior.

O monitoramento e controle foram realizados diariamente pela equipe administrativa operacional, semanalmente pela equipe médica e mensalmente pela coordenação e equipes multidsicplinares ao longo de dois anos. Acompanhando estes processos a Secretaria de Saúde do Estado de São Paulo.

Em relação ao Controle de Risco, e a demanda continental do SUS,19 o diagnóstico é de que, na área da Saúde, torna-se um desafio de grandes proporções a Análise de Risco com o "conforto" de que todas as variáveis foram equacionadas.

O Encerramento ocorreu após a inscrição dos primeiros doentes em lista junto a Secretaria de Saúde do Estado; o paciente JCL, que receberá um transplante Multivisceral e o paciente LLCA, o que representa que todos os processos do projeto foram concluídos e que o início do Programa de Transplante de Intestino e Multivisceral pode ser realizado. 
Tabela 1 - Análise segundo o $P M B O K 5^{\circ}$ edição, foram definidas as áreas de conhecimento e os respectivos Grupos de Processos, avaliação de cada correlação.

\begin{tabular}{|c|c|c|c|c|c|c|}
\hline $\begin{array}{c}\text { Áreas de } \\
\text { Conhecimento }\end{array}$ & Iniciação & Planejamento & Execução & $\begin{array}{l}\text { Monitoramento e } \\
\text { controle }\end{array}$ & Encerramento & Nota \\
\hline Integração & $\begin{array}{l}\text { Desenvolver } \\
\text { o termo de } \\
\text { abertura do } \\
\text { projeto } \\
++\end{array}$ & $\begin{array}{l}\text { Desenvolver o plano de } \\
\text { gerenciamento do projeto } \\
++\end{array}$ & $\begin{array}{l}\text { Orientar e gerenciar o } \\
\text { trabalho do projeto } \\
++\end{array}$ & $\begin{array}{l}\text { Monitorar e controlar } \\
\text { o trabalho do projeto } \\
1.5 \text {. Realizar o } \\
\text { controle integrado de } \\
\text { mudanças. } \\
++\end{array}$ & $\begin{array}{l}\text { Encerrar o } \\
\text { projeto ou fase } \\
+\end{array}$ & $\mathrm{A}$ \\
\hline Escopo & & $\begin{array}{l}\text { Planejar o Gerenciamento do } \\
\text { Escopo; } \\
\text { Coletar os requisitos; } \\
\text { Definir o escopo } \\
\text { Criar a EAP. } \\
++\end{array}$ & & $\begin{array}{l}\text { Validar o escopo } \\
\text { Controlar o escopo } \\
++\end{array}$ & & A \\
\hline Tempo & & $\begin{array}{l}\text { Planejar o gerenciamento do } \\
\text { Cronograma; } \\
\text { Definir as atividades; } \\
\text { Sequenciar atividades; } \\
\text { Estimar os recursos das } \\
\text { atividades; } \\
\text { Estimar as durações das } \\
\text { atividades; } \\
\text { Desenvolver o cronograma } \\
++.\end{array}$ & & $\begin{array}{l}\text { Controlar o } \\
\text { cronograma } \\
++\end{array}$ & & A \\
\hline Custos & & $\begin{array}{l}\text { Planejar o gerenciamento dos } \\
\text { Custos } \\
\text { Estimar custos } \\
\text { Determinar o orçamento } \\
++\end{array}$ & & $\begin{array}{l}\text { Controlar os custos } \\
++\end{array}$ & & A \\
\hline Qualidade & & $\begin{array}{l}\text { Planejar o gerenciamento da } \\
\text { qualidade } \\
++\end{array}$ & $\begin{array}{l}\text { Realizar a garantia de } \\
\text { qualidade } \\
++\end{array}$ & $\begin{array}{l}\text { Controlar a qualidade } \\
++\end{array}$ & & $\mathrm{A}$ \\
\hline $\begin{array}{l}\text { Recursos } \\
\text { Humanos }\end{array}$ & & $\begin{array}{l}\text { Planejar o gerenciamento dos } \\
\text { recursos humanos } \\
++\end{array}$ & $\begin{array}{l}\text { Mobilizar a equipe do } \\
\text { projeto } \\
\text { Desenvolver a equipe do } \\
\text { projeto } \\
\text { Gerenciar a equipe do } \\
\text { projeto } \\
++\end{array}$ & & & A \\
\hline Comunicações & & $\begin{array}{l}\text { Planejar o gerenciamento das } \\
\text { comunicações } \\
++\end{array}$ & $\begin{array}{l}\text { Gerenciar as } \\
\text { comunicações } \\
++\end{array}$ & $\begin{array}{l}\text { Controlar as } \\
\text { comunicações } \\
++\end{array}$ & & $\mathrm{A}$ \\
\hline Riscos & & $\begin{array}{l}\text { Planejar o gerenciamento dos } \\
\text { riscos; } \\
\text { Identificar os riscos; } \\
\text { Realizar a análise qualitativa dos } \\
\text { riscos; } \\
\text { Realiz.ar a análise quantitativa } \\
\text { dos riscos; } \\
\text { Planejar as respostas aos riscos. } \\
+\end{array}$ & & & & B \\
\hline Aquisição & $\begin{array}{l}\text { Identificar } \\
\text { partes } \\
\text { interessadas } \\
++ \\
\end{array}$ & $\begin{array}{l}\text { Planejar o gerenciamento das } \\
\text { partes interessadas } \\
++\end{array}$ & $\begin{array}{l}\text { Gerenciar o envolvimento } \\
\text { das partes interessadas } \\
++\end{array}$ & $\begin{array}{l}\text { Controlar o } \\
\text { envolvimento das } \\
\text { partes interessadas } \\
++\end{array}$ & & $\mathrm{A}$ \\
\hline $\begin{array}{l}\text { Partes } \\
\text { Interessadas }\end{array}$ & $\begin{array}{l}\text { Identificar } \\
\text { partes } \\
\text { interessadas } \\
++\end{array}$ & $\begin{array}{l}\text { Planejar o gerenciamento das } \\
\text { partes interessadas } \\
++\end{array}$ & $\begin{array}{l}\text { Gerenciar o envolvimento } \\
\text { das partes interessadas } \\
++\end{array}$ & $\begin{array}{l}\text { Controlar o } \\
\text { envolvimento das } \\
\text { partes interessadas } \\
++\end{array}$ & & A \\
\hline \multicolumn{7}{|c|}{$\begin{array}{ll}\text { Notas: } & \text { A - Relação forte da teoria com a realidade } \\
& \text { B - Relação fraca da teoria com a realidade } \\
& \text { EAP: Estrutura Analítica do Projeto }\end{array}$} \\
\hline
\end{tabular}




\section{CONCLUSÃO}

A análise, segundo os conhecimentos adquiridos, e a avaliação dos resultados de cada item com a nota final, demonstraram que durante o processo, os grupos de trabalho em cada processo e as suas áreas de atuação correlacionaram-se adequadamente conforme as necessidades ao longo do Projeto.

\section{ABSTRACT}

Purpose: To analyze with an established methodology Project Management Body of Knowledge (PMBOK) the implementation of an Intestine Transplant Service at HC-USP, in has a partner project between the National Transplant System (SNT) and NHS. Materials and Methods: Through the methodological analysis tool, the Project Management Body of Knowledge (PMBOK - Table 1) conducted the study in each stage of the Project Implementation (Program), with a critical look on each stage (Integration, Scope, Time, Cost, Quality, Human Resources, Communications, Risk, Procurement and Stakeholders). To analyze if the necessary steps have been performed in the correct way (those already performed) and to identify the areas for improvement in those tasks which have not yet been performed. Group Processes: 1 Initiation 2 Planning 3 Execution 4 Monitoring and Control 5 Closure. Results: The results were analyzed by using the concepts described in the Table. Conclusion: According to the knowledge acquired and the evaluation results of each item with a final note, the analysis has shown that along the process, the working groups in each process and their areas of operation were appropriately correlated, as it was required along the project. Table: According to the PMBOK 5th edition knowledge areas, the analysis has proven that each process and its areas of actuation were adequately correlated along the project.

Keywords: Organ Transplantation, Health Management, Intestines

\section{REFERÊNCIAS:}

1. Abu-Elmagd K, Fung J, Reyes J, Rao A, Jain A, Mazariegos G, et al. Hepatic and intestinal transplantation at the University of Pittsburgh. Clin Transpl. 1998:263-86.

2. Abu-Elmagd KM, Costa G, Bond GJ, Soltys K, Sindhi R, Wu T, et al. Five Hundred Intestinal and Multivisceral Transplantations at a Single Center: Major Advances With New Challenges. Ann Surg. 2009.

3. Andersen DA, Horslen S. An analysis of the long-term complications of intestine transplant recipients. Prog Transplant. 2004;14:277-82.

4. Bakonyi Neto A, Takegawa B, Ortolan E, Galvao F, Mendonca F, Sbragia L, et al. Demographic of short gut syndrome: increasing demand is not followed by referral of potential candidates for small bowel transplantation. Transplant Proc. 2004;36:259-60.

5. Fishbein TM. Intestinal transplantation. N Engl J Med 2009; 361:998-1008.

6. Goulet O, Revillon Y, Jan D, Brousse N, De Potter S, CerfBensussan N, et al. Small-bowel transplantation in children. Transplant Proc. 1990;22:2499-500.

7. Tannuri U. [Short bowel syndrome in children--treatment with home parenteral nutrition]. Rev Assoc Med Bras. 2004;50:3307.

8. Kato T, Tzakis AG, Selvaggi G, et al. Intestinal and multivisceral transplantation in children. Annals of Surgery. 2006;243:756-64; discussion 764-6.

9. Mazariegos GV, Soltys K, Bond G, Girnita A, Machaidze Z, Jaffe $R$, et al. Pediatric intestinal retransplantation: techniques, management, and outcomes. Transplantation. 2008;86:1777-82.
10.Mazariegos GV, Steffick DE, Horslen S, Farmer D, Fryer J, Grant D, et al. Intestine transplantation in the United States, 1999-2008. Am J Transplant. 2010;10:1020-34.

11.DiMartini A, Rovera GM, Graham TO, Furukawa H, Todo $S$, Funovits $M$, et al. Quality of life after small intestinal transplantation and among home parenteral nutrition patients. JPEN J Parenter Enteral Nutr. 1998;22:357-62.

12.O’Keefe SJ, Emerling M, Koritsky D, Martin D, Stamos J, Kandil $\mathrm{H}$, et al. Nutrition and quality of life following small intestinal transplantation. Am J Gastroenterol. 2007;102: 1093-100.

13. Rovera GM, Di Martini A, Graham TO, Hutson WR, Furukawa $\mathrm{H}$, Todo S, et al. Quality of life after intestinal transplantation and on total parenteral nutrition. Transplant Proc. 1998;30:2513-4.

14.Sudan D, Horslen S, Botha J, Grant W, Torres C, Shaw B, Jr., et al. Quality of life after pediatric intestinal transplantation: the perception of pediatric recipients and their parents. Am J Transplant. 2004;4:407-13.

15.Galvao FH. Transplante de Intestino e Multivisceral. In: Waitzberg DL, editor. Nutrição oral, enteral e parenteral na prática clínica. S. Paulo: Atheneu, 2008:1539-50.

16.Galvao FH, Waitzberg DL, Bacchella T, Gama-Rodrigues J, Machado MC. Small intestine transplantation. Arq Gastroenterol. 2003;40:118-25.

17. Galvão FHF. Transplante intestinal. In: Moraes IN, editor. Tratado de Clínica Cirúrgica. São Paulo: ROCA, 2005:2080-85.

18.A guide to the project management body of knowledge $\left(\mathrm{PMBOK}^{\odot}\right.$ guide). -- Fourth edition.

19. Malik AM, Vecina-Neto G. Gestão Em Saúde. Editora: São Paulo: Guanabara Koogan, 2011 (8527717085). 